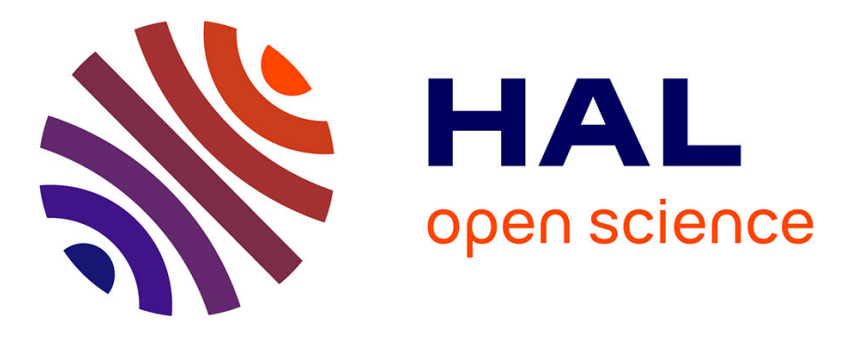

\title{
Automated landmarking for insects morphometric analysis using deep neural networks
}

Van-Linh Le, Marie Beurton-Aimar, Akka Zemmari, Alexia Marie, Nicolas

Parisey

\section{- To cite this version:}

Van-Linh Le, Marie Beurton-Aimar, Akka Zemmari, Alexia Marie, Nicolas Parisey. Automated landmarking for insects morphometric analysis using deep neural networks. Ecological Informatics, 2020, 60, 10.1016/j.ecoinf.2020.101175 . hal-03319822

\section{HAL Id: hal-03319822 \\ https://hal.inrae.fr/hal-03319822}

Submitted on 1 Sep 2021

HAL is a multi-disciplinary open access archive for the deposit and dissemination of scientific research documents, whether they are published or not. The documents may come from teaching and research institutions in France or abroad, or from public or private research centers.
L'archive ouverte pluridisciplinaire HAL, est destinée au dépôt et à la diffusion de documents scientifiques de niveau recherche, publiés ou non, émanant des établissements d'enseignement et de recherche français ou étrangers, des laboratoires publics ou privés. 


\title{
Automated Landmarking for Insects Morphometric Analysis using Deep Neural Networks
}

\author{
Le Van Linh ${ }^{\mathrm{a}, \mathrm{c}, *}$, Beurton-Aimar Marie ${ }^{\mathrm{a}, 1}$, Zemmari Akka ${ }^{\mathrm{a}}$, Marie Alexia ${ }^{\mathrm{b}}$, Parisey Nicolas ${ }^{\mathrm{b}, 1}$ \\ ${ }^{a}$ LaBRI - University of Bordeaux, UMR 5800, 351, cours de la Liberation, 33405 Talence, France \\ ${ }^{b}$ UMR 1349 IGEPP, BP 35327, 35653 Le Rheu, France \\ ${ }^{c}$ INRIA Bordeaux Sud-Ouest, 200, avenue de la Vieille Tour, 33405 Talence, France
}

\begin{abstract}
Landmarks are one of the important concepts in morphometry analysis. They are anatomical points that can be located consistently (e.g., corner of the eyes) and used to establish correspondence or divergence among morphologies of biological or non-biological specimens. Currently, the landmarks are mostly positioned manually by entomologists on numerical images. In this work, we propose a method to automatically predict the landmarks on entomological images based on Deep Learning methods, more specifically by using Convolutional Neural Network (CNN). We propose a CNN architecture, EB-Net, which is built in a modular way the concept of "Elementary Blocks", each made up of usual layer types of CNN. After using a custom data augmentation procedure, the network has been trained and tested on a data set of different anatomical part of carabids (pronotum, head and elytra). In this numerical experiment, we have generated two strategies to evaluate the network and to improve the obtained results: training from scratch or applying a fine-tuning step. The predicted landmark coordinates have been compared to the coordinates of the manual landmarks provided by the biologists. The statistical analysis of the distances between predicted and manual coordinates has shown that our predictions can replace efficiently manual landmarking and allows to propose automatization of such operation.
\end{abstract}

Keywords: Landmarks, Morphometry, Deep learning, Convolutional Neural Network

\section{Introduction}

In the context of ecosystem services, there is an interest in examining complex interactions between the evolution of insect populations and environmental factors affecting their functions. In order to assess specifically pestregulating services and in line with studies pointing to shape traducing function [1, there are more and more 5 researches about beneficial insect morphometrics 2, 3. In such morphometric studies, it is common to analyze subject's shape independently of their poses and sizes [4]. Since the late $20^{t h}$ century [5], rooted in a strong statistical background, geometric morphometrics address the study of such biological shapes [6]. It is an effective set of methods with several specialised softwares readily available [7,8. Geometric morphometrics uses a configuration of landmarks to describe shape, open curve or outline; and a landmark being a two or three-dimensional anatomically-relevant point. In order to investigate the possibility to automatize landmarking on beneficial insects, we chose to focus on

\footnotetext{
${ }^{*}$ Corresponding author

Email addresses: van-linh.le@labri.fr (Le Van Linh), beurton@labri.fr (Beurton-Aimar Marie), zemmari@labri.fr (Zemmari Akka), alexia.marie@inrae.fr (Marie Alexia), nicolas.parisey@inrae.fr (Parisey Nicolas)

${ }^{1}$ both authors contributed equally to this work.
} 
one of the most common and ubiquitous beneficial insect of north-western France, Poecilus cupreus (Carabidae). It is considered a polyphagous predator [9] beneficial to agriculture, being able to consume a large variety of agricultural pests including weed seeds, slugs and aphids [10]. As a Coleoptera, its morphological variability is usually measured on exoskeleton structures such as the head, pronotum and elytra (see Figure 2] [11.

Commonly, the first step in any morphometric geometrics study is the digital imaging of the biological specimens with controlled illumination and contrasting background. As such, morphometric landmark detection and positioning can be thought as a particular problem of features detection and solved using robust digital image processing [12. In the recent years, the term "deep learning" emerged describing class of computational models composed of multiple processing layers learning representations of data with multiple levels of abstraction [13]. Each layer extracts the representation of the input data from the previous layer and computes a new representation for the next layer. In the hierarchy of model, the lower layers take care of the primary features whereas the higher layers care for the abstract features to enlarge the aspect of input for the computational task (classification, regression, etc.) and to suppress irrelevant variations. Deep learning algorithms have proved to be very efficient in a wide variety of domains, notably computer vision [14, 15, 16, 17, 18, speech recognition [19, 20, 21], question answering [22] and language translation 23, 24. Within deep learning, Convolutional Neural Networks (CNNs) are well known for their success in many computer vision tasks such as image classification [14, 15] and objects recognition [17, 18. Recent success of this algorithm in human biometry [25] lead us to believe in its potential for insect morphometrics.

\subsection{Related works}

Landmark-based geometric morphometrics has been applied to a variety of research questions and applications in biology. The applications can be ranged from fossil human/dinosaurs 26, 27] to butterfly/fly wings [28, zebrafish skeletogenesis [29], flower shapes [30. They have been also concerned on medical imaging, e.g., cephalometry aims at analyzing the human cranium for orthodontic diagnosis and treatment planning [31, 32].

Geometric morphometry analysis based on landmarks is mainly beginning by positioning the landmarks in two-dimensional images, which is typically achieved manually. Landmarks are then compared by employing various statistical methods to distinguish landmark variations or the changing of shape in large populations, e.g., Procrustes analysis. Depending on applications, the number of landmarks varies, it could be ranged from several to dozens of landmarks, for example, 15 landmarks have been defined in a study on drosophila wing [33] or 25 landmarks have been used in a research on zebrafish [29, 34. Manual setting landmarks is time-consuming and difficult to reproduce. A solution that can automatically provide landmarks could be useful in these studies.

Recently, automatic prediction of landmarks has appeared in many applications of various domains: In computer vision, landmark localization is usually studied on human faces where we identify some points corresponding to significant parts on face, e.g., nose, eyes, mouth region [35, 36. In biology, the landmark identification problem has been appeared in the studies to analyse shape and size on the organisms [33, 37, e.g., analyzing the corolla shape variation. In biomedical field, the problem of automatic landmark positioning has been addressed in cephalometry

45 [32, 38. The familiar methods in these domains are based on the combination of template matching and prior knowledge information after a step of the segmentation of interesting objects. Lowe et al. 39] have proposed SIFT method to find the corresponding keypoints between two images. Palaniswamy et al. 33. have proposed a method 
based on probabilistic Hough Transform to automatically identify the landmarks in digital images of Drosophila wings. In previous work [40, we have proposed a method which was extended from Palaniswamy's method, to determine landmarks on beetle images. The experiments have been done on two sets of mandibles images which have an ordinary shape and are easy to segment. The obtained results were satisfying when comparing to the landmark's coordinates of the manual setting. Unfortunately, this method could not be provided the landmarks to other parts of beetles as pronotum, head, and elytra. The reasons have been found down that these pictures are not simply as mandible ones. They do not only contain the considered objects but also other parts of beetle because they have been captured before dissection. Also, shape segmentation has been a trap for our method.

In recent years, deep learning has been widely used in computer vision. Using Convolutional Neural Network (CNN) to determine the landmarks on 2D images has achieved good results. As was common, the CNN inputs raw pixels of the image, then it analyzes the relations between the pixels to predict the coordinates of landmarks. These operations are performed by a sequence of layers. It is worth to note we do not recognize the appearance of the segmentation step in the process. Thus, CNN has offered an effective solution to face images that have difficulty in segmentation. In the landmarking context, Yi Sun et al. [41] have proposed cascaded convolutional neural networks (three-levels) to predict the facial points of interest on the human face. Each level considers the face from global to local regions to determine the landmarks. Zhanpeng Zhang et al. 42] proposed a Tasks-Constrained Deep Convolutional Network to optimize facial landmarks detection. The model determines the facial landmarks with a set of related tasks such as head pose estimation, gender classification, age estimation, face recognition, or facial attribute inference. Cintas et al. 25] has introduced a network to predict the landmarks on human ears. After training, the network has the ability to predict 45 landmarks on human ears. In biology field, Porto et al. 43. have proposed a machine-learning-based high-throughput pipeline to collect high-dimensional morphometric data in two-dimensional images of semi-rigid biological structures. Based on our knowledge, CNNs have been widely used in biological applications but not to provide the landmarks. In this work, we proposed a CNN architecture to predict the landmarks on biological images, specific beetle's images.

\subsection{Contributions}

In this article, we detail a CNN architecture that we have designed to automatically set landmarks on beetle images, so-called Elementary Block Network (EB-Net). The prediction has been evaluated by comparing to the ground truth manually provided by biologists. We describe how we have applied data augmentation to remedy the problem of using machine learning algorithms on the small dataset. We will also outline how performance can be improved by using transfer learning from another dataset like human facial points.

\section{Material and Methods}

In this section, we first present the dataset that we have used in this study, as well as the strategies to pre-process the data. Then, we describe the designed network architecture to predict the landmarks in the beetle images.

\subsection{Dataset and preprocessing}

In order to provide the experimental data, we have selected the Brittany lands (North-West of France) to collect the samples. After collecting in three months, a collection of 293 beetles has been established (147 males and 146 
females/ 155 organic and 138 conventional agriculture) (Figure 1). As usual, images of beetles have been chosen to be studied instead of using real objects for practical reasons. For each beetle, five images corresponding to five parts of beetles have been taken into account: elytra, pronotum, head, left and right mandibles. The pictures of each body parts were captured under a trinocular magnifier at $\approx 300$ pixels per 1 millimeter (pixels/mm) for elytra, $\approx 600$ pixels $/ \mathrm{mm}$ for pronotum and head, 1500 pixels $/ \mathrm{mm}$ for mandibles. One can note that the head, pronotum, and elytra parts have been captured before dissection. The left and right mandibles have been separated from the beetle's body before taking the photos. All the images have been taken with the same camera under same conditions to release in the RGB color mode with a size of $3264 \times 2448$ pixels.

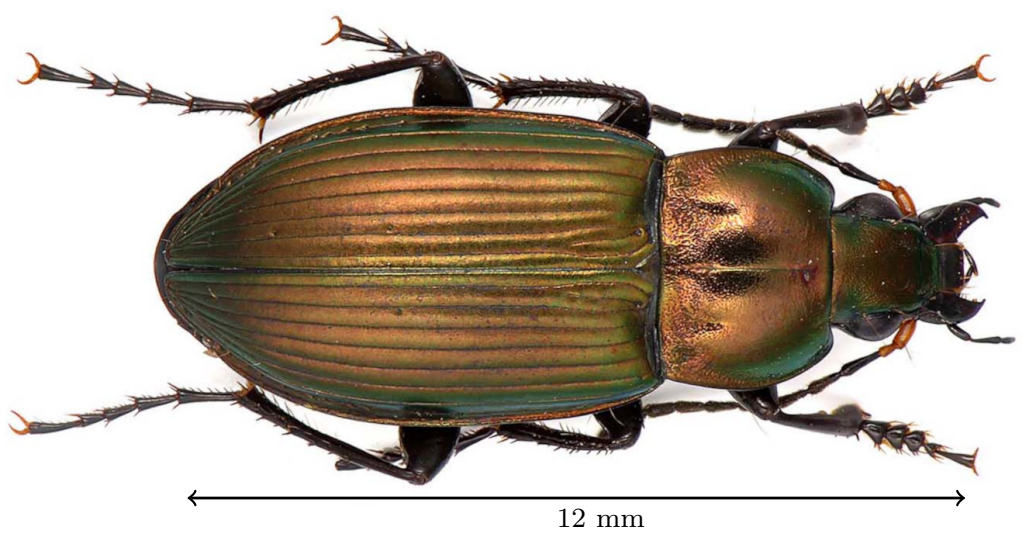

Figure 1: An illustration of the beetle.

In the next step, morphological landmarks were first set manually on the dorsal views of each body part of the beetles (head, pronotum, elytra, right and left mandibles). The morphology of each body part was processed and analyzed separately in order to limit variation resulting from their relative positions due to articulation. Landmarks were chosen according to the ease and the precision of their location on each specimen (Figure 2). Replicability analyses were performed to confirm the accuracy of landmarks positioning. They were positioned on each picture with TPSDig2 software (version 2.17) (Rohlf, 2013a). In some individuals, mandibles could not be processed because they were lacking or missing. For each specific part, a set of number of landmarks has been provided, for example, 8 landmarks for pronotum, 10 landmarks for head, 11 landmarks for elytra, 16 and 18 landmarks for left and right mandibles, respectively (Figure 22. In the context of this study, these manual landmarks have been used as ground truth to evaluate the output of our method.

The success stories [14, 15, 16, have proved that CNN models have to be trained on a large dataset with an enormous number of data samples before using the trained model to perform on testing data. Training the model with a big dataset can help the model able to learn more different cases and to improve the learning ability of the network. Unfortunately, providing a large dataset is too costly or even impossible in several domains, e.g., in biology, archaeology, medicine. However, a dataset has less than one thousand images is a typical size, and it is possible to do Deep Learning on such sample size thanks to data augmentation. In our case, we have only 293 images for each part of the beetles. This number is large from the point of view of manual operations, but it is far from the thousand images. So, we have applied data augmentation to face this problem. 


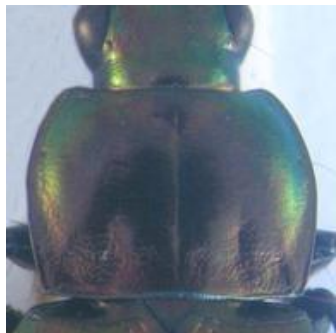

(a) . Pronotum.

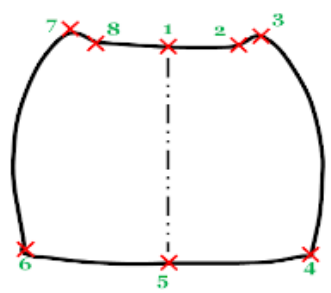

(f) . Pronotum.

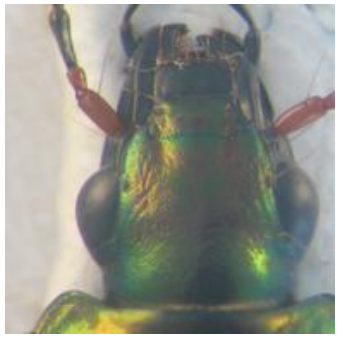

(b) . Head.

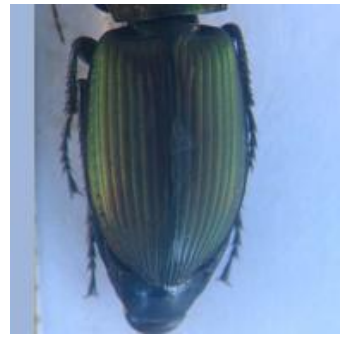

(c) . Elytra.

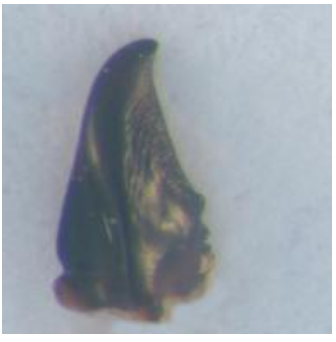

(d) . Left mandible.

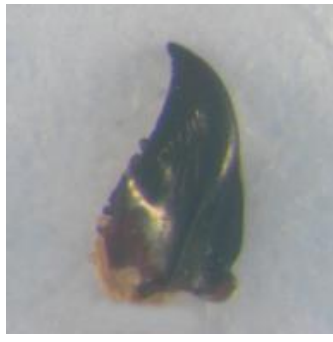

(e) . Right mandible.

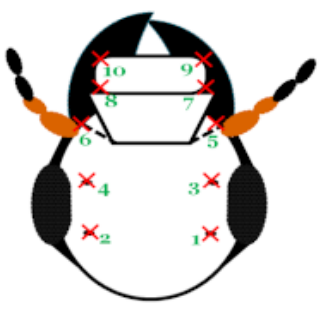

(g) . Head.

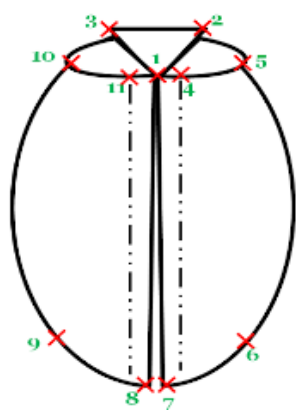

(h) . Elytra.

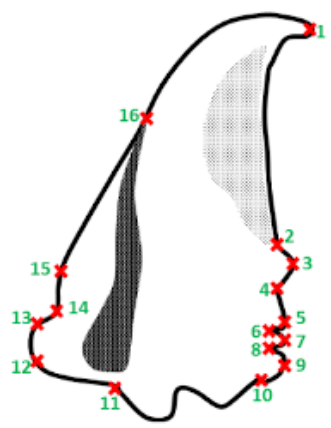

(i) . Left mandible.

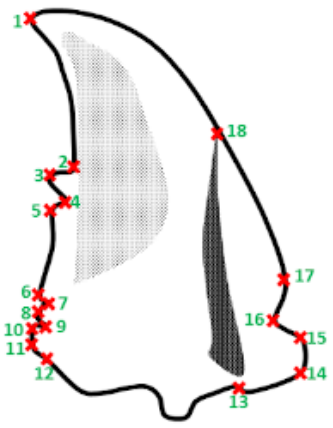

(j) . Right mandible.

Figure 2: The sample images in our dataset (top), the 5 parts and their landmarks defined manually by biologists (bottom).

Most often in deep learning applications, dataset augmentation uses operations such as translation, rotation, scaling, shearing or cropping, which are well-known efficient to generate new versions of existing images [14, 44]. However, it exists some CNNs that are inherently invariant to rotation/translation/ scaling, etc. [44]. In order to select the right method for our application, we have done some tests by moving the object in the image. In each time, we have quickly gone to the over-fitting in the training step. Consequently, we have preferred other ways to produce misshapen images by operating on the image's color channels. We have proposed two strategies to augment the number of images as inputs for our model.
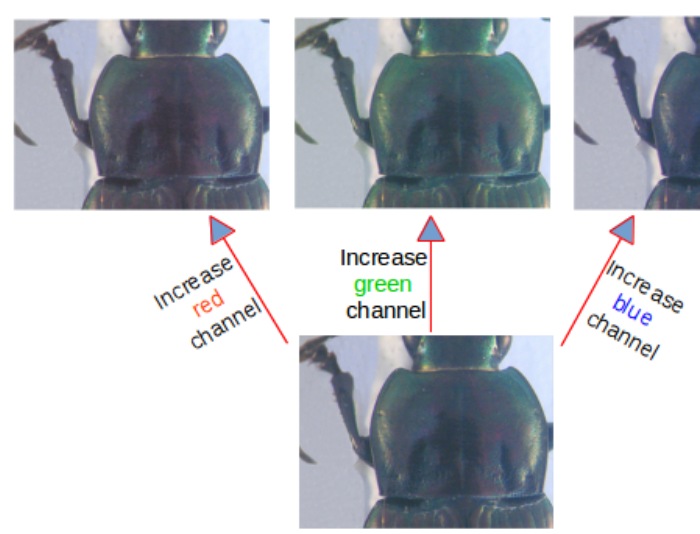

(a) . Add a constant to each channel.
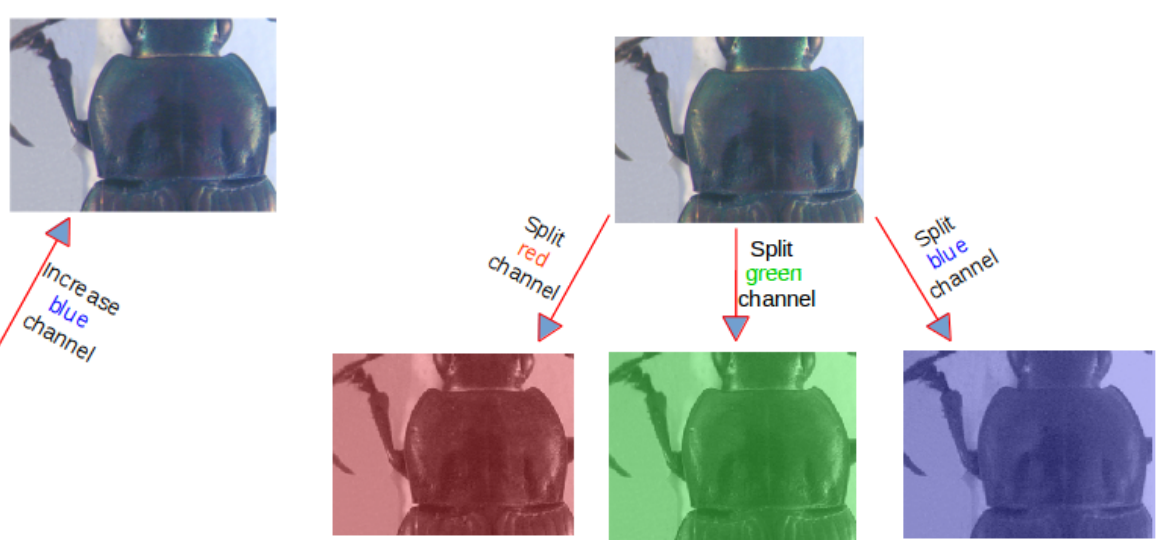

(b). Split the channels of image.

Figure 3: The two strategies to augment the number of images in pronotum set.a) illustrate the augmentation channel per channel by adding a constant value to each pixel on the corresponding channel. b) illustrate how to extract 3 different images from only one by selecting a color channel each time. 
The first strategy has been applied to change the value of each channel in the original image. According to this, a constant have been added to a channel of RGB image for each time. We chose a constant over a random draw from a distribution so that we always add a sufficient disturbance over the original image but not too much disturbance so that the network don't learn "noise". For example, if we add a constant $c=10$ to the red channel from an original RGB image, we will obtain a new image with the values at red channel by greater than the red channel of original image a value of 10. By this way, we can generate three new RGB images from a RGB image.

The second procedure was to split the channels of RGB images in order to create three gray-scale images. This work seems promising because the network model works on single-channel images. At the end, we have generated six versions from an image. In total, we have obtained $293 \times 7=2051$ images for each set of images. Figure 3 illustrates the two described strategies.

To perform the objective, we have observed the input size of the several CNN models [14, 15, 25, 41, and noticed that most often their input sizes were limited to 256 pixels. One can note that our images were released with the size of $3264 \times 2448$, as mentioned in Section 2.1. This size is a bit heavy for training the network. Consequently, we have down-sampled (scaled) our images to a new size of $256 \times 192$ to respect the ratio between width and height. Of course, coordinates of landmarks have been down-sampled to the new size of images. Practically, convergence is usually faster if the average of each input variable over the training set is close to zero [45]. So, the brightness of the image is normalized to $[0,1]$.

\subsection{Network architecture}

As mentioned in Section 1. CNNs are well-known for tasks on computer vision. The layers parameters which usually use to set up the layers in a CNN, as well as the hyper-parameter setting could be easy found in the literature [13, 46] or in the short glossary ${ }^{2}$. Our initial trials were inspired by AlexNet architecture [14. The model has been designed by combining in sequence the classical layers, e.g., convolutional (CONV), max pooling (POOL) and fully-connected (FC) layers. Unfortunately, over-fitting effects appearance very quickly. In deep learning, Dropout (DROP) layer is well-known to prevent over-fitting [47] and usually used in the last steps of the procedures. We will present now the Elementary Block (EB) model which includes all these layer types and is the core of our model architecture.

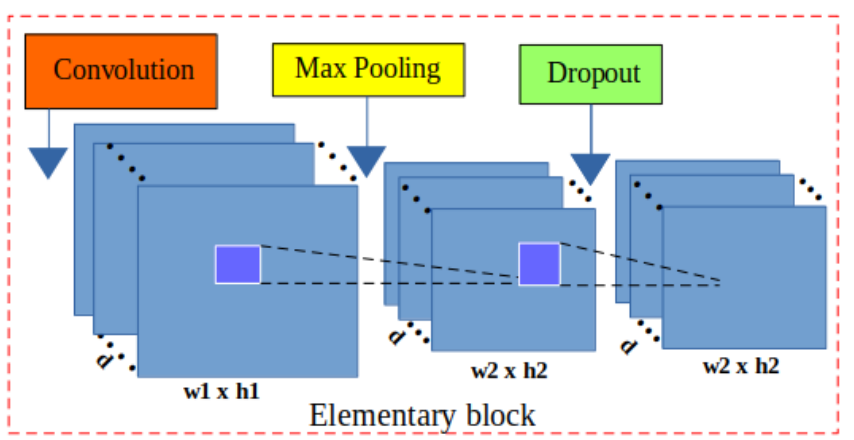

Figure 4: The components of an Elementary Block. Orange, yellow and green boxes contain the operations, blue boxes are the data.

\footnotetext{
${ }^{2}$ https://developers.google.com/machine-learning/glossary
} 
Figure 4 illustrates the order of the layers in an EB item. It is defined as a sequence of a CONV layer, a maximum POOL layer, and a Dropout layer. In an Elementary Block, the convolution layer is used to extract the high-level features by applying different filters on the input. Setting POOL layer after the CONV layer towards to reduce the dimension of the representation to decrease the number of parameters and also reduce the computing time. The DROP layer, usually inserted between the FC layers to prevent over-fitting [13, 14, is included in our model in the extracting feature blocks to produce some kinds of image noise augmentation. The DROP layer randomly drops some connections during the training process. It makes the network thinner than the original one (fewer parameters), so training a network with dropout layer is equivalent to train a set of thinner networks.

Three Elementary Blocks have been then assembled to create the whole network architecture, called Elementary Blocks Network (EB-Net). The used parameters of layers in EBs have been set as follows: the depths of CONV layers are set to $(32,64,128)$ with a small kernel $(3 \times 3,2 \times 2,2 \times 2)$ from the first to the third block, respectively. The POOL layers in all three blocks have been designed with a filter of $2 \times 2$ and a stride of 2 pixels. With these parameter values, the spatial size of the image will be halved after every block. The probabilities of the dropout layers are set increasing: $0.1,0.2$, and 0.3 , respectively. In order to extract the global relationship between the features and to provide the prediction, three fully-connected layers have been added after the combination of three EBs. The first FC layer takes all features from the last block as the input for computing. The last FC layer outputs the coordinates prediction of landmarks. The number of outputs at each FC layers is set to 1000,1000, and $X$, respectively. The number $X$ is equal to twice the number of landmarks that we want to predict $(x, y$ coordinates). For example, to predict 8 landmarks on pronotum, the number of outputs of the last FC layer is set at 16. Additionally, a dropout layer with the probability equals to 0.5 has been inserted between the first and the second fully-connected layers as usual [4]. Figure 5 resumes the EB-Net architecture.

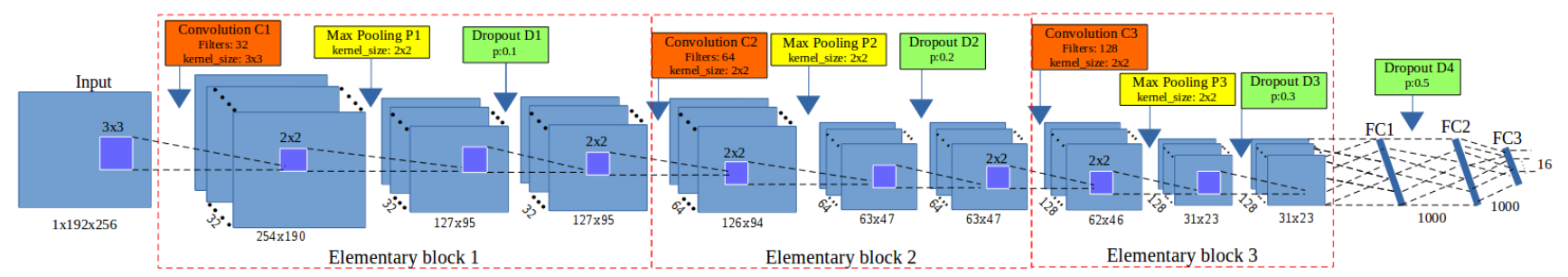

Figure 5: Elementary Blocks Network (EB-Net) architecture obtained from a composition of 3 Elementary Blocks and ended by 3 Fully-Connected layers.

In a CNN model, besides the model-specific parameters which involve the structure of the network (e.g., the number of layers or parameter values of each layer), the optimized hyper-parameters are also important to design a CNN model. These variables are related to the training of the network model, for example, the loss function, the number of epochs, the batch size, and the initialized value of learning rate. Practically, the range of the possible values is explored through empirical observations depending on the task and the dataset. In our application, we have chosen the most common optimization algorithm, Stochastic Gradient Descent (SGD) combined with backpropagation. In order to apply SGD, two relevant parameters need to be provided: learning rate and momentum. In our application, the learning rate begins from 0.03 and stops at 0.00001 , and the momentum rate is updated from 0.9 to 0.9999 . During the training, learning rate and momentum are adjusted to fit with the number of epochs. For 
example, the learning rate and momentum at the first/the third epoch are 0.03/0.029994 and 0.9/0.90001, respectively. Besides, the Root Mean Squared Error (RMSE) has been chosen as loss function because it is employed for regression problems where outputs represent quantitative values as in the case of coordinates of landmarks. The EB-Net has been implemented by using Lasagne framework [49, 50], and trained in 5000 epochs on Linux system by using a NVIDIA GPU (Titan X) card.

\subsection{Setting and training EB-Net}

In order to provide the predicted landmarks for all images, we have applied cross-validation technique K-fold with $k=9$, to select the test images. We will call a selection step, a round. For each round, we take 260 images for training and validation processes, the 33 remaining images will be kept for testing step and these images are never seen by the model. It is worth to note that the set of 260 images has been augmented by the strategies described in Section 2.1, to provide $1820(260 \times 7)$ images for training and validation steps. The cross-validation step has been achieved after 9 rounds in total.

During the training and validation step, the 1820 images are randomly divided into training and validation sets with a ratio of $60 \%: 40 \%$. At each training step, the pair of image and its manual landmarks is inputted to train the network model. In the testing step, we input the image only to the trained model in order to predict landmarks. In our case, the manual landmarks have been given by the biologists. So, they are used as ground truth to train the network, as well as to evaluate the predicted ones.

\subsection{Statistical evaluation of predicted shapes}

The quality of landmarks predicted by the network must be assessed in view of the desired usage for these predictions. It is quite frequent in geometric morphometrics to use the landmarks for subsequent Procrustes regression [51] which is a method to quantify the relative amount of shape variations (among specimens) attributable to one or more factors in a linear model. Before the regression step, a generalized Procrustes analysis [51] translates all specimens related landmarks to the origin, scales them to unit-centroid size, and optimally rotates them until the coordinates of corresponding points align at best. The resulting aligned coordinates represent the shape of each specimen. Afterward, the Procrustes distance between specimens (i.e., sum-of-squared distance between corresponding landmarks) can be used for regression purpose [52. The crucial step that we can take to ensure the validity of using predicted landmarks for Procrustes regression is a measure of shape covariation between predicted and manual landmarks [53]. This problem is related to the construction of a latent variable of shape deformation, i.e., estimating how landmarks move as a group. Of course, this goes further than studying, independently, each landmark correlation between predicted versus manual. At the very least it means we are interested, for $L$ landmarks, in the whole $L \times L$ correlation matrix not just in its diagonal.

\section{Results}

\subsection{The first evaluation}

EB-Net has been firstly trained and tested on the pronotum images. Figure 6 shows the losses of training and validation processes in one round on pronotum images. The blue curve is the training loss, and the green curve 
is the validation one. As we can notice, learning is effective and over-fitting does not appear because validation loss reaches to the same performance as training loss. We can see that EBs with the help of dropout layers have properly worked to prevent the over-fitting.

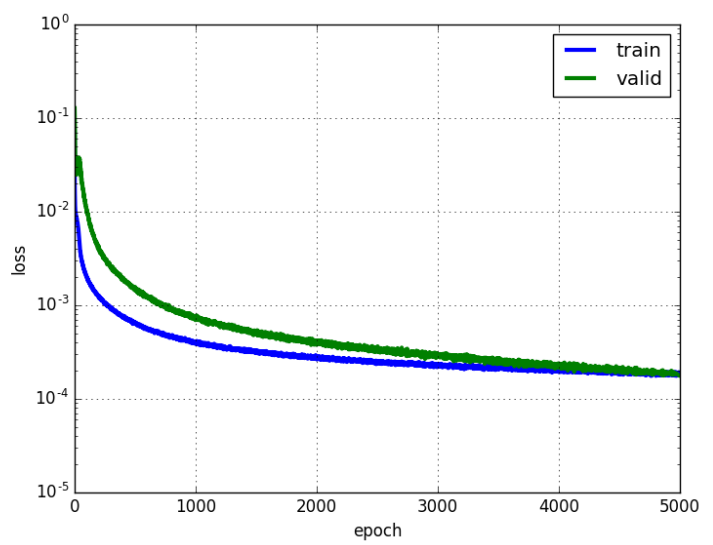

Figure 6: The losses during training EB-Net on pronotum images.

As mentioned, we have used RMSE as loss value. It was computed based on the distances between manual and predicted landmarks, so we tried to minimize the RMSE values during the training and validation processes. Table 1 shows the losses of the 9 rounds of EB-Net training on pronotum images. We can observe that although the image dataset changes through each round, the loss values remain stable and always under $3 \times 10^{-3}$. Based on the success of EB-Net on pronotum images, we have employed it on the two sets of head and elytra images with the same result qualities.

\begin{tabular}{|c|c|c|c|c|c|c|c|c|c|}
\hline Round & 1 & 2 & 3 & 4 & 5 & 6 & 7 & 8 & 9 \\
\hline Training loss & 0.00018 & 0.00019 & 0.00019 & 0.00021 & 0.00021 & 0.00019 & 0.00018 & 0.00018 & 0.00020 \\
\hline Validation loss & 0.00019 & 0.00021 & 0.00026 & 0.00029 & 0.00029 & 0.00018 & 0.00018 & 0.00021 & 0.00027 \\
\hline
\end{tabular}

Table 1: The losses during the training of EB-Net on pronotum images

The performances of a CNN is most often measured from the loss or the accuracy. But in this work, we also need to appreciate correctly where the estimated landmarks are positioned by the network. So, the trained model of each round has been used to predict the landmarks in images of the corresponding testing set. Then, the coordinates of outputted landmarks are evaluated by comparing with the manual ones. Figure 7 illustrates the landmarks on the three parts of beetles. The red/yellow points present the predicted/manual landmarks. One can note that even some predicted landmarks are close to the manual ones, we have also some predicted coordinates that are far from the expected results.

In order to provide a global evaluation, the distances (in pixels) have been calculated between the manual landmarks and the corresponding predicted ones, and the average value at each position has been taken into account. Table 2 shows the obtained average distance for pronotum, head and elytra. With the image's size of $256 \times 192$, we can consider that an error around $1 \%$ of image size $(\approx 2$ pixels $)$ could be accepted. Unfortunately, 


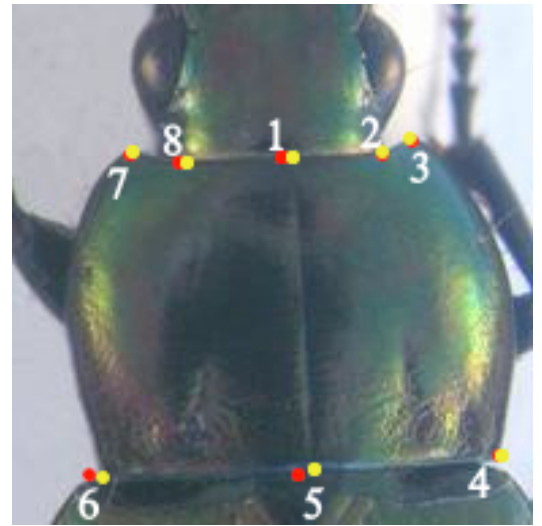

(a) . Pronotum

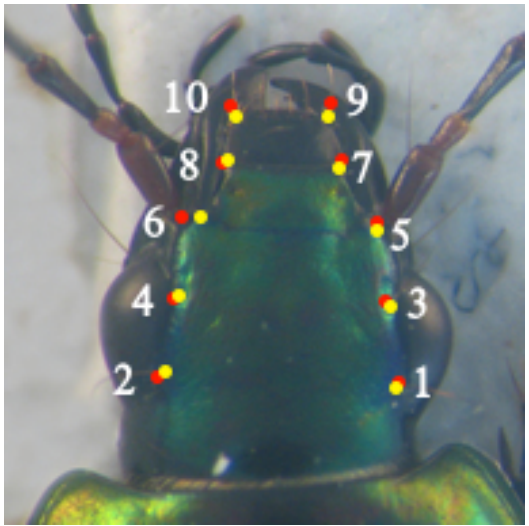

(b) . Head

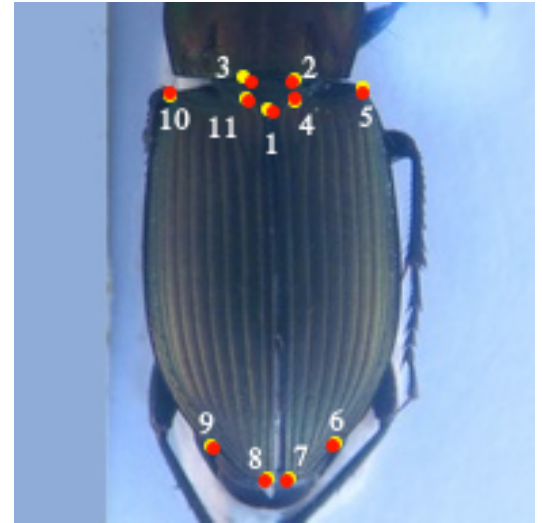

(c) . Elytra

Figure 7: The landmarks on three parts of beetle. The red/ yellow points present the predicted/ manual landmarks.

our results exhibit the average distances from 4 to 5 pixels $(\approx 2 \%$ of error).

\begin{tabular}{|c|c|c|c|c|c|c|c|c|c|c|c|}
\hline Landmark & 1 & 2 & 3 & 4 & 5 & 6 & 7 & 8 & 9 & 10 & 11 \\
\hline Pronotum & 4.00 & 4.48 & 4.3 & 4.39 & 4.29 & 5.36 & 4.64 & 4.94 & - & - & - \\
\hline Head & 5.53 & 5.16 & 5.38 & 5.03 & 4.84 & 4.45 & 4.79 & 4.53 & 5.14 & 5.06 & - \\
\hline Elytra & 3.87 & 3.97 & 3.92 & 3.87 & 4.02 & 4.84 & 5.21 & 5.47 & 5.27 & 4.07 & 3.99 \\
\hline
\end{tabular}

Table 2: The average distances per landmark on images of each set.

It is worth to note that an average value could reflect several different cases, for example, the values closed together (small dispersion) or two sets of values very far (large dispersion). In order to see in which situation we are, Figure 8 shows the distribution of distances between the manual and predicted landmarks of all images for the best and the worst average values (corresponding to the green and red values in the Table 2). Each point presents the distance between the points (landmarks) of an image. The lines (blue/red) illustrate the average distance in each case. In both of two cases (the best and the worst), the distances mostly stayed in the region from 0 to the average value. Regarding these figures, it is clear that even a large number of points remains inside the range from 0 to average value, some points are widespread between the average and 15 or 25 pixels. The question is: is it possible to lower the mean and to lower the dispersion above the mean value?

\subsection{Transfer learning to improve performances}

Working with deep learning generally requires not only to design a good architecture but also to provide a huge dataset to train and test the model. Practically, this is a potential problem in some application domains as in biology, and we have to augment the number of samples before using it to train the model. However, the augmented dataset is far away from several hundred thousand images. In this case, knowledge transfer or transfer learning between tasks is another solution to deal with this problem, and it could be an additional solution to improve the prediction.

Transfer learning is a technique in deep learning to re-purpose a model which has been designed for a specific task (source task) on another related task (target task) [54, 55]. Choosing which strategy of transfer learning to 


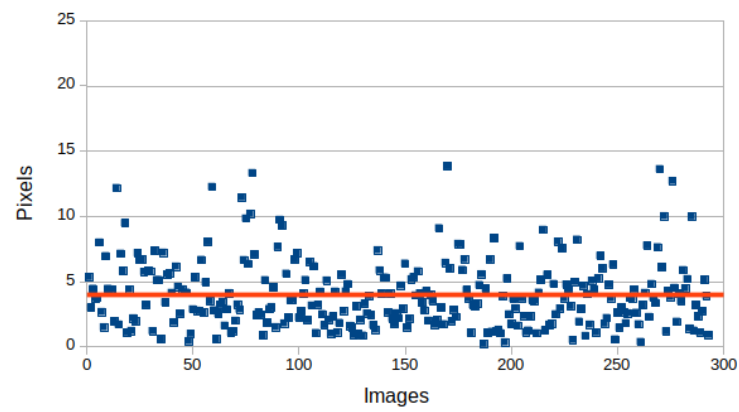

(a). The $1^{\text {st }}$ landmark (pronotum)

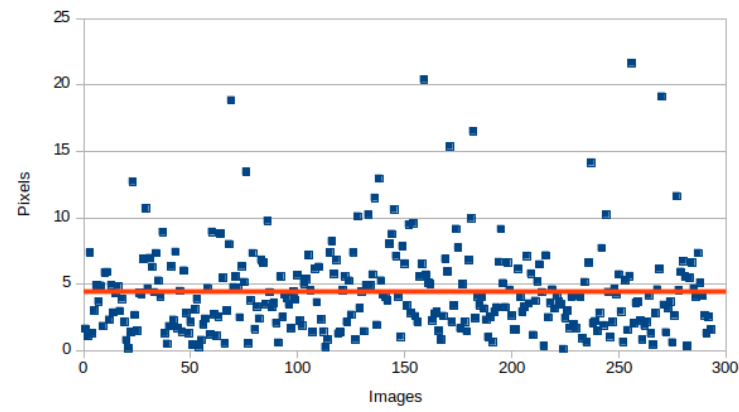

(c). The $6^{\text {th }}$ landmark (head)

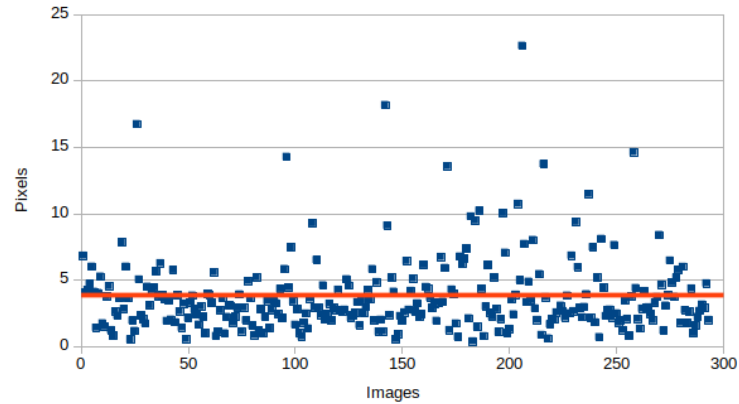

(e). The $1^{\text {st }}$ landmark (elytra)

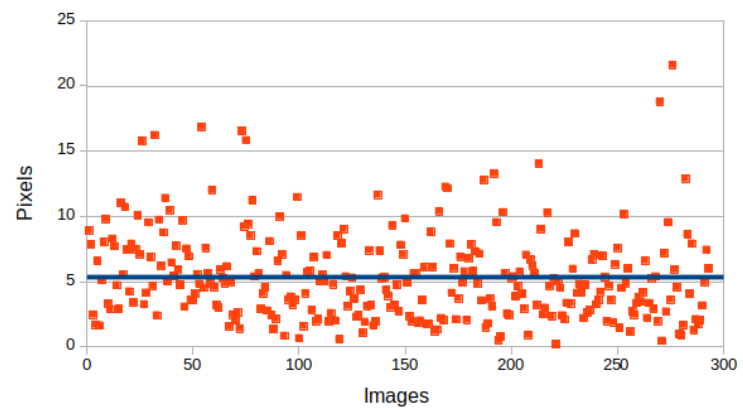

(b). The $6^{\text {th }}$ landmark (pronotum)

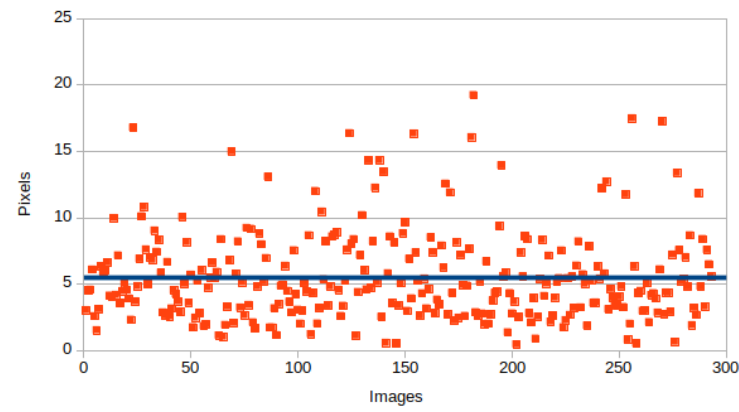

(d). The $1^{\text {st }}$ landmark (head)

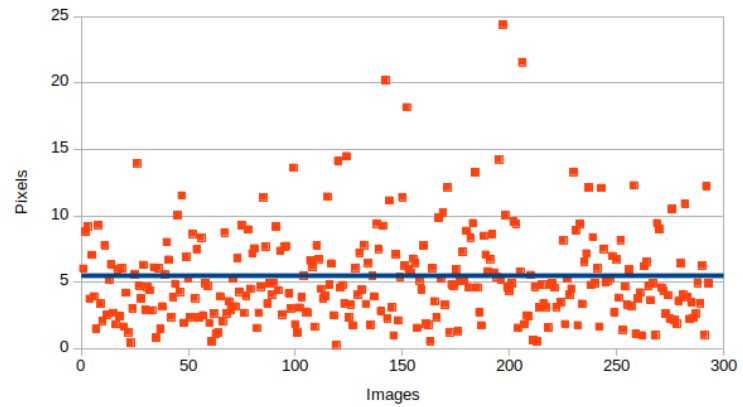

(f). The $8^{\text {th }}$ landmark (elytra)

Figure 8: The distribution of distances for the best and the worst cases of the three parts

apply depending on the relationship between two tasks, as well as the size of database. Practically, transfer learning is often targeted on 2 strategies:

- Use CNN as a fixed feature extractor: Taking a CNN pre-trained on a large dataset, then removing the last (or several) fully-connected layers and using the rest layers of CNN as a fixed extractor for the new dataset.

- Fine-tuning a CNN: This strategy replaces the fully-connected layers (the last one or some layers) as the first strategy has done. However, it does not only replace and retrain the last layer but also fine-tunes the weights of the pre-trained model by extending the backpropagation. One can note that to reuse a pre-trained model, the parameters have to be adapted between the two tasks. These parameters could be the size of input images, the number of outputs, or the parameters of each layer.

In deep learning community, ImageNet is known as a large dataset with more than 14 million images of thousands 
of categories [56]. Notable models have employed ImageNet as training data to solve different tasks [14, 57]. These pre-trained models have been widely shared in deep learning community as a source to re-use the features of 2140 images of human faces $(96 \times 96$ pixels $)$. For each image, 15 landmarks have been defined: 6 landmarks for eyes, 4 landmarks for eyebrows, 4 landmarks for the mouth, and 1 landmark for nose tip. Figure 9 shows 4 examples of face images and their landmark positions in the dataset.
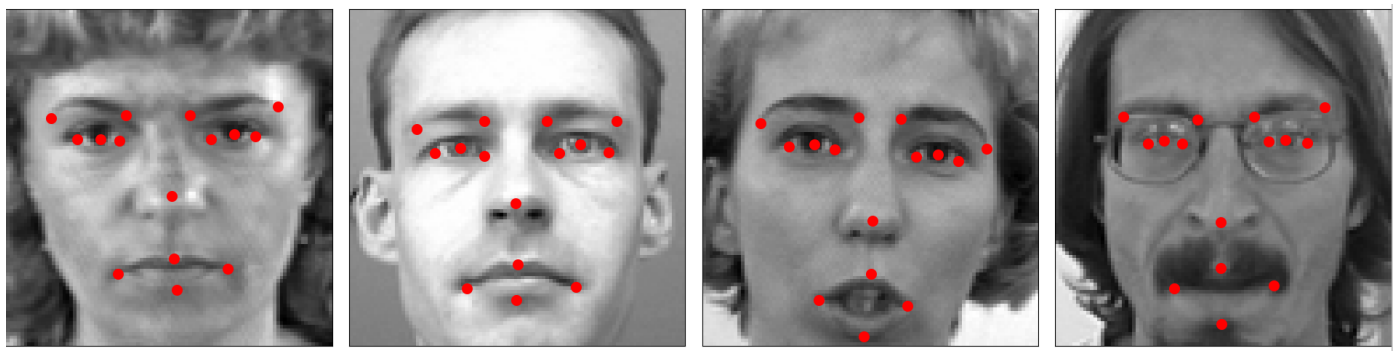

Figure 9: The samples (face and manual landmarks) in the dataset on Kaggle that we have used to pre-train EB-Net.

In order to use EB-Net on this dataset, we have adapted the parameters of the input and the output layers to match with the face images size and the landmark number. The new parameter values are $96 \times 96$ for the input size, and 30 for the number of outputs of the last FC layer (corresponding to 15 landmarks). For hyper-parameters, just the number of epochs have changed to reach the value of 10000 epochs. In table 3 , we show the RMSE score and the comparison of the effectiveness between EB-Net and the three best ones that have been published for the challenge.

\begin{tabular}{|c|c|c|c|c|}
\hline Team & Olegra & Trump & Enes & EB-Net \\
\hline RMSE score (in pixels) & 1.2824 & 1.4004 & 1.4026 & $\mathbf{1 . 4 9 7}$ \\
\hline
\end{tabular}

Table 3: RMSE comparison between our score and top three of challenge.

\footnotetext{
${ }^{3}$ Deepfake Detection Challenge/ Facial Keypoints Detection

${ }^{4}$ https://www.kaggle.com/c/facial-keypoints-detection
} 
It is worth to note that the three scores have been obtained by testing the models on a private set of images that are not freely available. To do the test, we have had to use some images getting from the public data set, 100 images have been chosen to do that. Comparing with these scores, the three models present better results than ours but we are very close. The RMSE score is around 1 pixel that is an acceptable error to display the landmarks on the images. Consequently, the pre-training of EB-Net is considered as correct and we have decided to re-use the pre-trained parameters values to fine-tune the model for beetle images.

\subsubsection{Fine-tuning on beetle images}

As we have mentioned, fine-tuning is a strategy of transfer learning that could boost the efficiency of a model on a target task. Technically, the weights of a CNN model can be fine-tuned by continuing the backpropagation, and it exists two ways to perform fine-tuning process: frozen and unfrozen.

- Frozen scenario: the parameters of lower layers (close to the input layer) will be fixed, we fine-tune only the higher ones (close to the output layer).

- Unfrozen scenario: allows continuing to update the parameter values of all layers in the model.

In order to fine-tune EB-Net on beetle images, we have gone with unfrozen process to continue updating the parameter values. One can note that the sizes of images in the two datasets are different: the beetle images have a size of $256 \times 192$ pixels; whereas the size of facial images is $96 \times 96$ pixels, far from the beetle images. Consequently, adjustments are needed to match the two tasks.

First of all, reducing the resolution of the beetle images to $96 \times 96$ could be lead to a loss of essential information. As our images contain a background band, it is easy to suppress it with a pre-processing operation, we have chosen to remove a part of the background region (without any beetle's parts) instead of down-sampling our pictures. So, the new beetle images are finally set to $192 \times 192$ pixels. The EB-Net parameters will be settled to take into account the differences of the values between the pre-training and fine-tuning steps. To declare the modification, we set the stride value of the first convolutional layer to 2 (as the usual way to do [55]).

\subsubsection{Fine-tuning results}

We present all the obtained results for the three beetle parts: pronotum, head and elytra, in the same way than in the previous results in order to provide an explicit comparison. Tables 4 , 5, 6 show the comparison at each position between the average distances provided by the two processes (training from scratch and fine-tuning) on pronotum, head, and elytra, respectively. The first row presents the landmark number; From scratch row reminds the previously average distances when EB-Net has been trained from scratch; Fine-tune row presents the new average distances; the last row presents the improvement percentage between the two processes. The green and red values are respectively the best and the worst values in each process. All distances are given in pixel unity. In all these tables, all the average distances have decreased between 1 and 1.5 pixels in both of three sets of images. Clearly, the fine-tuning process has improved the landmark prediction for each group. The best-predicted positions (green ones) have changed but it exists a group of well-predicted landmarks in each set of images, such as:

- For pronotum: the $1^{\text {st }}, 3^{\text {rd }}$, and $7^{\text {th }}$ landmark. 
- For head: the $6^{\text {th }}, 7^{\text {th }}, 8^{\text {th }}$, and $10^{\text {th }}$ landmark.

- For elytra: the $1^{\text {st }}-5^{\text {th }}, 10^{\text {th }}$, and $11^{\text {th }}$ landmark.

At the opposite side, the worst cases remain the same positions as previously: the $6^{\text {th }}, 1^{\text {st }}$, and $8^{\text {th }}$ landmark on pronotum, head, and elytra, respectively.

\begin{tabular}{|c||c|c|c|c|c|c|c|c|}
\hline Landmark & 1 & 2 & 3 & 4 & 5 & 6 & 7 & 8 \\
\hline \hline From scratch & 4.00 & 4.48 & 4.3 & 4.39 & 4.29 & 5.36 & 4.64 & 4.94 \\
\hline Fine-tune & 2.99 & 3.41 & 2.98 & 3.54 & 3.37 & 4.06 & 2.93 & 3.64 \\
\hline \% of impr. & 25.25 & 24.01 & 30.56 & 19.18 & 21.55 & 24.28 & 36.85 & 26.16 \\
\hline \hline Variance & 3.27 & 4.99 & 4.26 & 5.92 & 5.16 & 6.70 & 3.94 & 6.16 \\
\hline IR (Interquartile Range) & 2.11 & 2.52 & 2.16 & 2.81 & 2.45 & 2.96 & 2.45 & 3.10 \\
\hline Min & 0.23 & 0.17 & 0.22 & 0.16 & 0.1 & 0.17 & 0.23 & 0.16 \\
\hline Max & 14.25 & 18.41 & 19.21 & 22.89 & 17.46 & 14.07 & 14.09 & 15.46 \\
\hline
\end{tabular}

Table 4: Average distances comparison on pronotum images and other scores. The landmarks number 1 and 7 remain the best estimation after fine-tunning, the number 6 is the worst one.

\begin{tabular}{|c||c|c|c|c|c|c|c|c|c|c|}
\hline Landmark & 1 & 2 & 3 & 4 & 5 & 6 & 7 & 8 & 9 & 10 \\
\hline \hline From scratch & 5.53 & 5.16 & 5.38 & 5.03 & 4.84 & 4.45 & 4.79 & 4.53 & 5.14 & 5.06 \\
\hline Fine-tune & 4.82 & 4.21 & 4.73 & 4.11 & 4.18 & 3.5 & 3.92 & 3.4 & 4.17 & 3.94 \\
\hline \% of impr. & 12.83 & 18.43 & 12.15 & 18.42 & 13.69 & 21.43 & 18.29 & 24.94 & 18.88 & 22.01 \\
\hline \hline Variance & 8.55 & 8.62 & 8.51 & 8.48 & 7 & 8.05 & 6.4 & 6.47 & 6.43 & 7.26 \\
\hline IR & 3.91 & 2.94 & 3.73 & 2.89 & 3.25 & 2.71 & 2.84 & 2.65 & 2.89 & 2.65 \\
\hline Min & 0.37 & 0.21 & 0.27 & 0.19 & 0.27 & 0.24 & 0.21 & 0.15 & 0.44 & 0.15 \\
\hline Max & 21.18 & 23.94 & 19.12 & 21.65 & 20.23 & 22.68 & 21.03 & 21.23 & 22.03 & 20.72 \\
\hline
\end{tabular}

Table 5: Average distances comparison on head images and other scores. The landmarks number 6 and 8 obtain the best estimation and the number 8 is the worst one.

Considering on each landmark position, the level of improvement is different depending on the difficulty of its location (surrounding area). But, all cases have been improved even they are the best or the worst cases. With the help of fine-tuning, the predictions have gained from $36.85 \% / 24.94 \% / 18.36 \%$ to $19.18 \% / 12.15 \% / 12.82 \%$ on pronotuom, head, and elytra, respectively.

As we have mentioned before, the mean value hides different situations. So, we have checked again these distribution of distances. Figure 10, 11, 12, shows the distribution of distances on two samples in each set of images: pronotum, head, and elytra, respectively. In these charts, the x-axis and y-axis present the image number (id) and the distance (in pixel). Each point in the chart represents a distance between manual and predicted landmarks, respectively. The blue/red lines in charts present the average values. We can observe from the figures that the 


\begin{tabular}{|c||c|c|c|c|c|c|c|c|c|c|c|}
\hline Landmark & 1 & 2 & 3 & 4 & 5 & 6 & 7 & 8 & 9 & 10 & 11 \\
\hline \hline From scratch & 3.87 & 3.97 & 3.92 & 3.87 & 4.02 & 4.84 & 5.21 & 5.47 & 5.27 & 4.07 & 3.99 \\
\hline Fine-tune & 3.21 & 3.28 & 3.2 & 3.22 & 3.31 & 4.21 & 4.54 & 4.76 & 4.55 & 3.39 & 3.29 \\
\hline \% of impr. & 17.04 & 17.34 & 18.36 & 16.61 & 17.66 & 13.13 & 12.82 & 12.96 & 13.69 & 16.68 & 17.54 \\
\hline \hline Variance & 9.39 & 10.26 & 9.03 & 9.62 & 10.24 & 11.23 & 12.3 & 11.93 & 10.75 & 9.2 & 9.11 \\
\hline IR & 2.2 & 2.33 & 2.37 & 2.32 & 2.38 & 2.82 & 3.2 & 3.54 & 3.46 & 2.19 & 2.07 \\
\hline Min & 0.13 & 0.16 & 0.08 & 0.15 & 0.12 & 0.21 & 0.35 & 0.47 & 0.27 & 0.18 & 0.05 \\
\hline Max & 32.67 & 34 & 31.09 & 33.15 & 35.8 & 35.3 & 34.74 & 32.17 & 28.35 & 29.92 & 32.36 \\
\hline
\end{tabular}

Table 6: Average distances comparison on elytra images and other scores. The landmarks number 1 and 3 obtain the best estimation and the number 8 is the worst one.

distance values are very different from the two processes (training from scratch and fine-tuning). The distances have been reduced with the help of fine-tuning process whatever they were the low or the high values in the case of training from scratch. We have gained more points (in the chart) in the range from zero to the average distance, and the number of extreme values have been decrease too. For example, in the worst case of pronotum (the $6^{\text {th }}$ landmark), we do not see any point greater than 15 pixels with the fine-tuning process. However, it exists some difficult cases that the model could not optimize in the head or the elytra parts. These cases could be noted as the outlier values in our dataset.

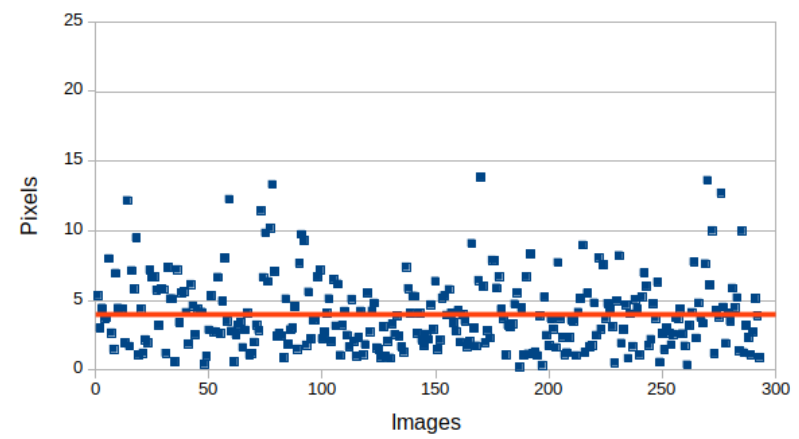

(a) $1^{\text {st }}$ landmark (from scratch)

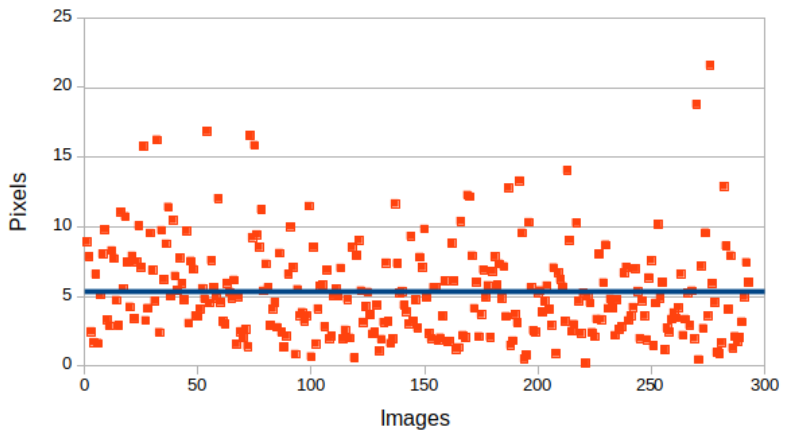

(c) $6^{\text {th }}$ landmark (from scratch)

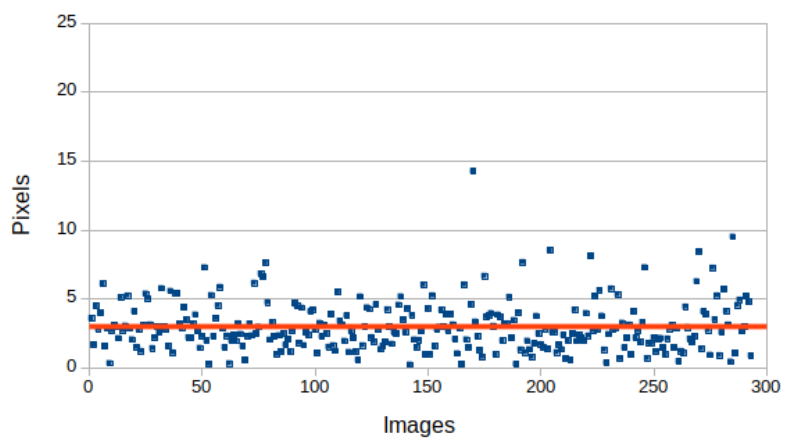

(b) $\cdot 1^{\text {st }}$ landmark (fine-tuning)

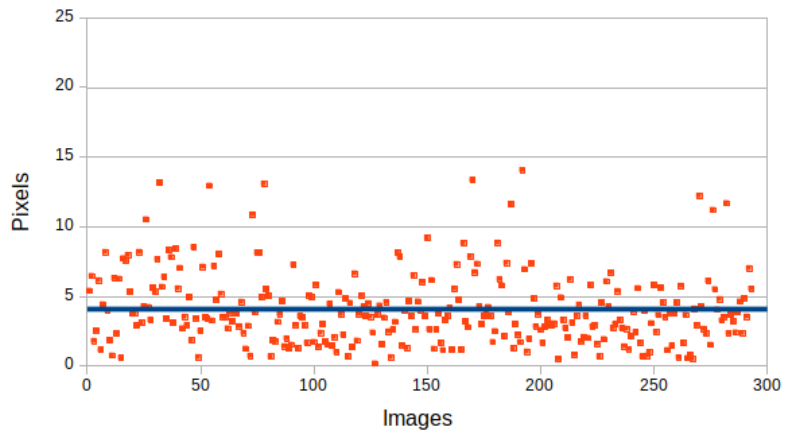

(d) $\cdot 6^{\text {th }}$ landmark (fine-tuning)

Figure 10: A comparison of distances distribution of the $1^{\text {st }}$ landmark and the worst case $\left(6^{\text {th }}\right.$ landmark) on pronotum images. 


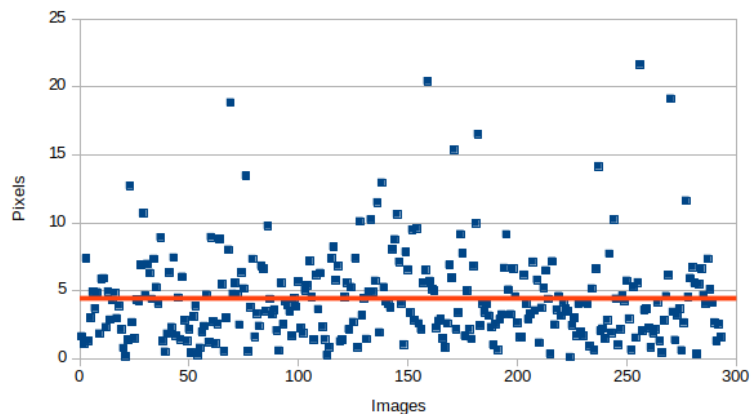

(a) $6^{\text {th }}$ landmark (from scratch)

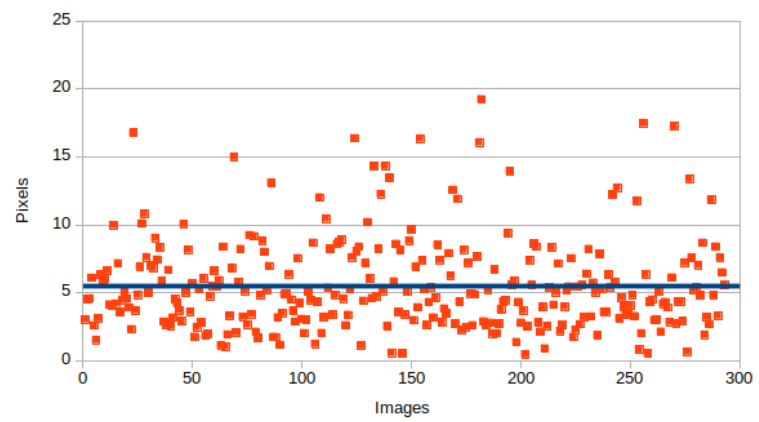

(c) $\cdot 1^{\text {st }}$ landmark (from scratch)

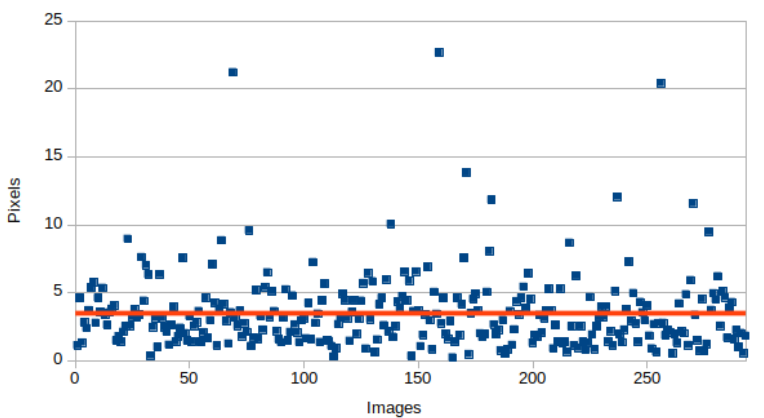

(b) $\cdot 6^{\text {th }}$ landmark (fine-tuning)

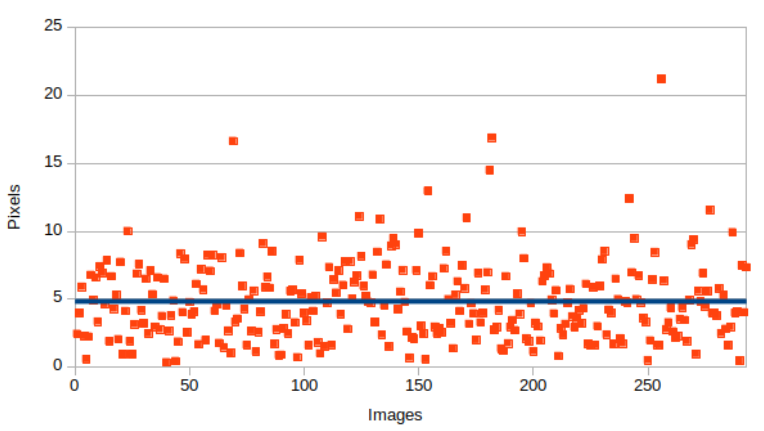

(d) $\cdot 1^{\text {st }}$ landmark (fine-tuning)

Figure 11: The distribution of distances of all head images on $1^{\text {st }}$ landmark and $6^{\text {th }}$ landmark.

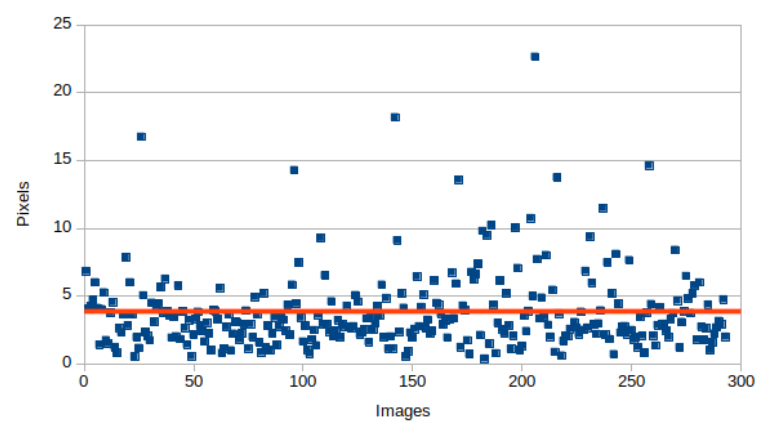

(a) $1^{\text {st }}$ landmark (from scratch)

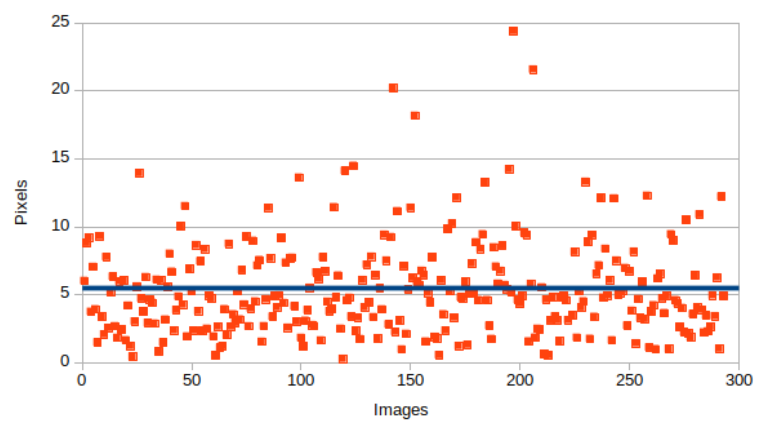

(c) $\cdot 8^{\text {th }}$ landmark (from scratch)

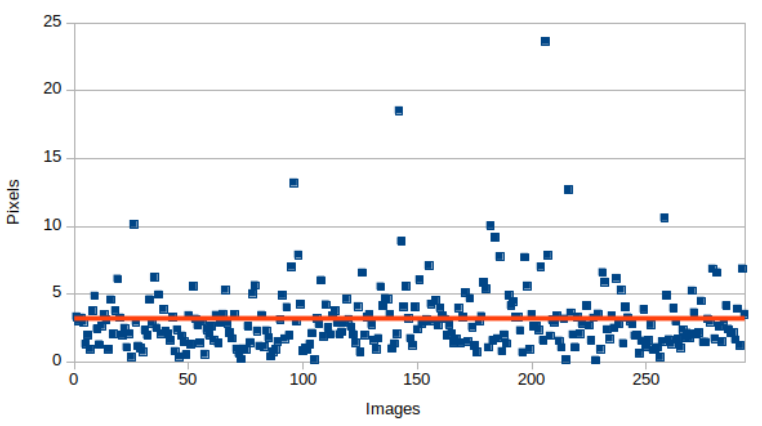

(b) $.1^{\text {st }}$ landmark (fine-tuning)

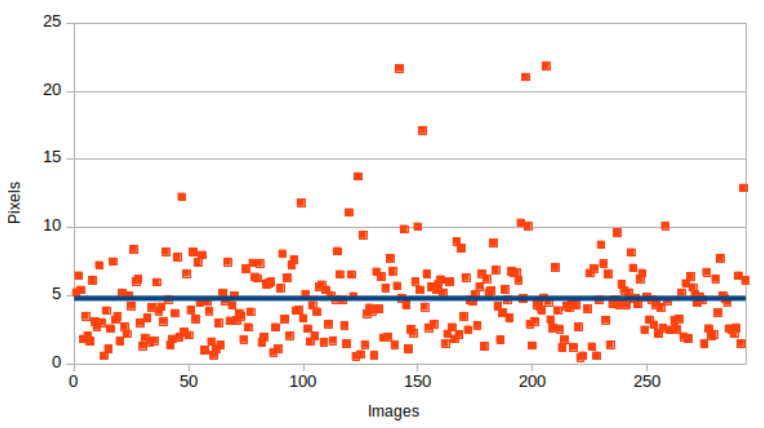

(d) $\cdot 8^{t h}$ landmark (fine-tuning)

Figure 12: The distribution of distances of all elytra images on $1^{\text {st }}$ landmark and $8^{\text {th }}$ landmark. 
To illustrate the results, Figure 13 shows both predicted (in red) and manual (in yellow) landmarks on three random images from the three sets of images. Clearly, the predictions have been improved, they are more closer to the manual ones.

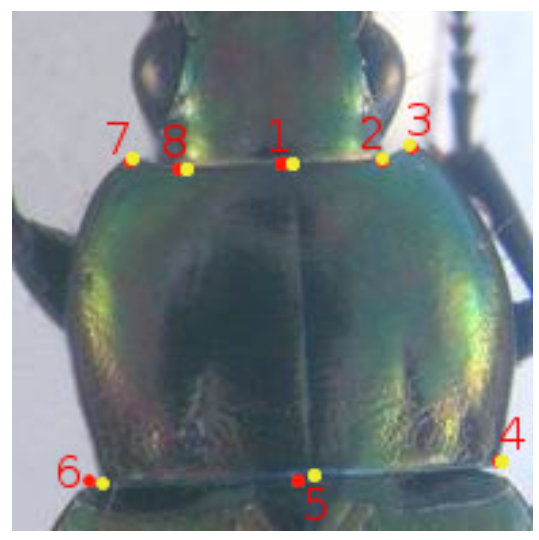

(a) . Pronotum

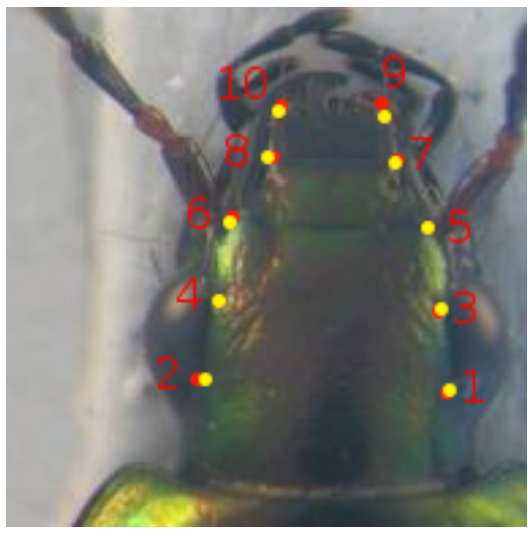

(b) . Head

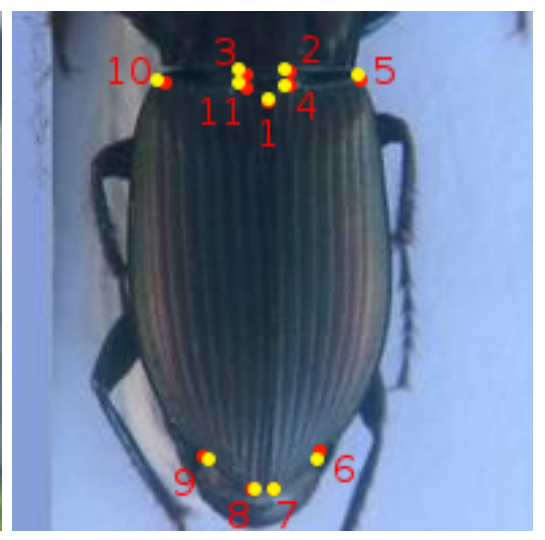

(c) . Elytra

Figure 13: The location of predicted/manual landmarks in one case of each part.

The red/yellow points represent the predicted/manual landmarks.

\subsubsection{Predicted shapes : comparing 'from-scratch' to fine-tuning}

We have measured shape covariations between predicted landmarks and manual ones [53, assessing the performance of fine-tuned and 'from scratch' networks (described previously) on each anatomical parts. The shape covariation is expressed as a correlation coefficient and an associated p-value.

\begin{tabular}{|c||c|c|c|c|}
\hline \multicolumn{1}{|c||}{} & \multicolumn{2}{c|}{ From scratch } & \multicolumn{2}{c|}{ Fine-tune } \\
\cline { 2 - 5 } & cor & p-value & cor. & p-v. \\
\hline Pronotum & 0.4118 & 0.001 & 0.7424 & 0.001 \\
\hline Head & 0.5145 & 0.001 & 0.7337 & 0.001 \\
\hline Elytra & 0.2513 & 0.407 & 0.3025 & 0.069 \\
\hline
\end{tabular}

Table 7: Shape covariation between predicted and manual landmarks: shape covariation as a correlation coefficient (cor.) and an associated p-values (p-v.)

We can see from table 7 that shape correlations varies between 0.2513 and 0.7424 and that 4 out of 6 of them are statistically significant (i.e., p-value $<0.001$ ). For all the anatomical parts, the fine-tuning improved the shape correlations and its statistical significance. Best results are obtained for fine-tuned networks predicting pronotum's and head's landmarks with high significant correlations, 0.7224 and 0.7337 respectively. Fine-tuning enhanced predictions on Elytra but not enough to make them usuable. We end up with a majority $\left(\frac{2}{3}\right)$ of anatomical parts where the predicted landmarks are good candidate for replacing manual ones in any procrustes regressions. 


\section{Discussion and perspectives}

In this article, we have presented a convolutional neural network, EB-Net, for automatically predict the landhave been slightly improved, but we have spent more resources and computing times to reach this improvement. The average distances have been improved by 0.5 pixels. However, this change was statistically insignificant (data not shown). Consequently, we need to note that it exists a balance between the depth of the model, the accuracy of outputs and the cost to compute. This elementary block approach is not, formally, a neural architecture search

In this paper, we focused on a carabid species which is linked to studies in agroecology and conservation ecology. But EB-Net has the potential to be applied to other insects and invertebrates as well. In [66], a bibliographic survey

\footnotetext{
${ }^{5}$ Source Web of Science ${ }^{T M}$ accessed on 04/24/2020.
} 
of articles published between 2000 and 2015 resulted in finding 472 publications that used geometric morphometric methods to describe the shape of insects, with a linear 20 fold increase of publications over the period and a

URL http://onlinelibrary.wiley.com/doi/10.1890/ES14-00075.1/abstract

[4] D. G. Kendall, The diffusion of shape, Advances in Applied Probability 9 (3) (1977) 428-430. doi:10.1017/S0001867800028743

URL https://www.cambridge.org/core/journals/advances-in-applied-probability/article/ diffusion-of-shape/7CFF1175D4DCCF6063E403847120BE7B

[5] F. L. Bookstein, Foundations of Morphometrics, Annual Review of Ecology and Systematics 13 (1) (1982) 451-470. doi:10.1146/annurev.es.13.110182.002315.

[6] F. J. Rohlf, On Applications of Geometric Morphometrics to Studies of Ontogeny and Phylogeny, Systematic Biology 47 (1) (1998) 147-158. doi:10.1080/106351598261094.

URL https://academic.oup.com/sysbio/article-lookup/doi/10.1080/106351598261094

\footnotetext{
6 https://github.com/linhlevandlu/CNN_Beetles_Landmarks
} 
口 [7] D. C. Adams, E. Otárola-Castillo, geomorph: an r package for the collection and analysis of geometric morphometric shape data, Methods in Ecology and Evolution 4 (4) (2013) 393-399. doi:10.1111/2041-210X.12035. URL http://onlinelibrary.wiley.com/doi/10.1111/2041-210X.12035/abstract

[8] C. P. Klingenberg, MorphoJ: an integrated software package for geometric morphometrics, Molecular Ecology Resources 11 (2) (2011) 353-357. doi:10.1111/j.1755-0998.2010.02924.x.

[9] A. Larochelle, The Food of Carabid Beetles:(coleoptera: Carabidae, Including Cicindelinae), Sillery: Association des entomologistes amateurs du Québec, 1990.

[10] B. Kromp, Carabid beetles in sustainable agriculture: a review on pest control efficacy, cultivation impacts and enhancement, Agriculture, Ecosystems \& Environment 74 (1) (1999) 187-228. doi:10.1016/S0167-8809(99) 00037-7.

URL http://www.sciencedirect.com/science/article/pii/S0167880999000377

${ }_{425}^{4}$ [11] T. Eldred, C. Meloro, C. Scholtz, D. Murphy, K. Fincken, M. Hayward, Does size matter for horny bee1. tles? A geometric morphometric analysis of interspecific and intersexual size and shape variation in Colophon haughtoniBarnard, 1929, and C. kawaii Mizukami, 1997 (Coleoptera: Lucanidae), Organisms Diversity \& Evolution 16 (4) (2016) 821-833. doi:10.1007/s13127-016-0289-z.

URL https://link.springer.com/article/10.1007/s13127-016-0289-z

${ }_{430}$ [12] R. C. Gonzalez, R. E. Woods, Digital Image Processing (3rd Edition), Prentice-Hall, Inc., Upper Saddle River, NJ, USA, 2006.

[13] Y. LeCun, Y. Bengio, G. Hinton, Deep learning, Nature 521 (7553) (2015) 436-444.

[14] A. Krizhevsky, I. Sutskever, G. E. Hinton, Imagenet classification with deep convolutional neural networks, in: Advances in neural information processing systems, 2012, pp. 1097-1105.

${ }_{435}$ [15] D. Ciregan, U. Meier, J. Schmidhuber, Multi-column deep neural networks for image classification, in: Computer Vision and Pattern Recognition (CVPR), 2012 IEEE Conference on, IEEE, 2012, pp. 3642-3649.

[16] C. Szegedy, W. Liu, Y. Jia, P. Sermanet, S. Reed, D. Anguelov, D. Erhan, V. Vanhoucke, A. Rabinovich, Going deeper with convolutions, in: Proceedings of the IEEE Conference on Computer Vision and Pattern Recognition, 2015, pp. 1-9.

${ }_{440}^{40}$ [17] H. Li, Z. Lin, X. Shen, J. Brandt, G. Hua, A convolutional neural network cascade for face detection, in: Proceedings of the IEEE Conference on Computer Vision and Pattern Recognition, 2015, pp. 5325-5334.

[18] J. J. Tompson, A. Jain, Y. LeCun, C. Bregler, Joint training of a convolutional network and a graphical model for human pose estimation, in: Advances in neural information processing systems, 2014, pp. 1799-1807.

[19] T. Mikolov, A. Deoras, D. Povey, L. Burget, J. Cernockỳ, Strategies for training large scale neural network 445 language models, in: Automatic Speech Recognition and Understanding (ASRU), 2011 IEEE Workshop on, IEEE, 2011, pp. 196-201. 
[20] G. Hinton, L. Deng, D. Yu, G. E. Dahl, A.-r. Mohamed, N. Jaitly, A. Senior, V. Vanhoucke, P. Nguyen, T. N. Sainath, et al., Deep neural networks for acoustic modeling in speech recognition: The shared views of four research groups, IEEE Signal Processing Magazine 29 (6) (2012) 82-97.

[21] T. N. Sainath, A.-r. Mohamed, B. Kingsbury, B. Ramabhadran, Deep convolutional neural networks for lvcsr, in: 2013 IEEE international conference on acoustics, speech and signal processing, IEEE, 2013, pp. 8614-8618.

[22] A. Bordes, S. Chopra, J. Weston, Question answering with subgraph embeddings, arXiv preprint arXiv:1406.3676.

[23] I. Sutskever, O. Vinyals, Q. V. Le, Sequence to sequence learning with neural networks, in: Advances in neural information processing systems, 2014, pp. 3104-3112.

[24] S. Jean, K. Cho, R. Memisevic, Y. Bengio, On using very large target vocabulary for neural machine translation, arXiv preprint arXiv:1412.2007.

[25] C. Cintas, M. Quinto-Sánchez, V. Acuña, C. Paschetta, S. de Azevedo, C. C. S. de Cerqueira, V. Ramallo, C. Gallo, G. Poletti, M. C. Bortolini, et al., Automatic ear detection and feature extraction using geometric morphometrics and convolutional neural networks, IET Biometrics 6 (3) (2016) 211-223.

[26] A. Rosas, L. Pérez-Criado, M. Bastir, A. Estalrrich, R. Huguet, A. García-Tabernero, J. F. Pastor, M. De la Rasilla, A geometric morphometrics comparative analysis of neandertal humeri (epiphyses-fused) from the el sidrón cave site (asturias, spain), Journal of human evolution 82 (2015) 51-66.

[27] J. L. Fearon, D. J. Varricchio, Morphometric analysis of the forelimb and pectoral girdle of the cretaceous ornithopod dinosaur oryctodromeus cubicularis and implications for digging, Journal of Vertebrate Paleontology 35 (4) (2015) e936555.

[28] N. Chazot, S. Panara, N. Zilbermann, P. Blandin, Y. Le Poul, R. Cornette, M. Elias, V. Debat, Morpho morphometrics: shared ancestry and selection drive the evolution of wing size and shape in morpho butterflies, Evolution 70 (1) (2016) 181-194.

[29] J. Aceto, R. Nourizadeh-Lillabadi, R. Marée, N. Dardenne, N. Jeanray, L. Wehenkel, P. Aleström, J. J. van Loon, M. Muller, Zebrafish bone and general physiology are differently affected by hormones or changes in gravity, PloS one 10 (6).

[30] T. van der Niet, C. P. Zollikofer, M. S. P. de León, S. D. Johnson, H. P. Linder, Three-dimensional geometric morphometrics for studying floral shape variation, Trends in plant science 15 (8) (2010) 423-426.

475 [31] C. Lindner, C.-W. Wang, C.-T. Huang, C.-H. Li, S.-W. Chang, T. F. Cootes, Fully automatic system for accurate localisation and analysis of cephalometric landmarks in lateral cephalograms, Scientific reports 6 (2016) 33581.

[32] V. Grau, M. Alcaniz, M. Juan, C. Monserrat, C. Knoll, Automatic localization of cephalometric landmarks, Journal of Biomedical Informatics 34 (3) (2001) 146-156. 
[33] S. Palaniswamy, N. A. Thacker, C. P. Klingenberg, Automatic identification of landmarks in digital images, IET Computer Vision 4 (4) (2010) 247-260.

[34] R. Vandaele, J. Aceto, M. Muller, F. Peronnet, V. Debat, C.-W. Wang, C.-T. Huang, S. Jodogne, P. Martinive, P. Geurts, et al., Landmark detection in 2d bioimages for geometric morphometrics: a multi-resolution treebased approach, Scientific reports 8 (1) (2018) 1-13.

[35] X. P. Burgos-Artizzu, P. Perona, P. Dollár, Robust face landmark estimation under occlusion, in: Proceedings of the IEEE international conference on computer vision, 2013, pp. 1513-1520.

[36] H. Yang, I. Patras, Sieving regression forest votes for facial feature detection in the wild, in: Proceedings of the IEEE International Conference on Computer Vision, 2013, pp. 1936-1943.

[37] Y. Savriama, A step-by-step guide for geometric morphometrics of floral symmetry, Frontiers in plant science 9 (2018) 1433.

[38] H. Mohseni, S. Kasaei, Automatic localization of cephalometric landmarks, in: 2007 IEEE International Symposium on Signal Processing and Information Technology, IEEE, 2007, pp. 396-401.

[39] D. G. Lowe, Distinctive image features from scale-invariant keypoints, International journal of computer vision 60 (2) (2004) 91-110.

[40] V. L. Le, M. Beurton-Aimar, A. Krähenbühl, N. Parisey, MAELab: a framework to automatize landmark estimation, in: WSCG 2017, 25th International Conference in Central Europe on Computer Graphics, Visualization and Computer Vision'2017, Plzen, Czech Republic, 2017.

URL https://hal.archives-ouvertes.fr/hal-01571440

[41] Y. Sun, X. Wang, X. Tang, Deep convolutional network cascade for facial point detection, in: Proceedings of the IEEE conference on computer vision and pattern recognition, 2013, pp. 3476-3483.

[42] Z. Zhang, P. Luo, C. C. Loy, X. Tang, Facial landmark detection by deep multi-task learning, in: European Conference on Computer Vision, Springer, 2014, pp. 94-108.

[43] A. Porto, K. L. Voje, Ml-morph: A fast, accurate and general approach for automated detection and landmarking of biological structures in images, Methods in Ecology and Evolution 11 (4) (2020) 500-512.

[44] C. Shorten, T. M. Khoshgoftaar, A survey on image data augmentation for deep learning, Journal of Big Data 6 (1) (2019) 60.

[45] Y. A. LeCun, L. Bottou, G. B. Orr, K.-R. Müller, Efficient backprop, in: Neural networks: Tricks of the trade, Springer, 2012, pp. 9-48.

[46] I. Goodfellow, Y. Bengio, A. Courville, Deep Learning, MIT Press, 2016, http://www.deeplearningbook.org.

[47] N. Srivastava, G. E. Hinton, A. Krizhevsky, I. Sutskever, R. Salakhutdinov, Dropout: a simple way to prevent neural networks from overfitting., Journal of machine learning research 15 (1) (2014) 1929-1958. 
[48] G. E. Hinton, N. Srivastava, A. Krizhevsky, I. Sutskever, R. R. Salakhutdinov, Improving neural networks by preventing co-adaptation of feature detectors, arXiv preprint arXiv:1207.0580.

[49] F. Bastien, P. Lamblin, R. Pascanu, J. Bergstra, I. Goodfellow, A. Bergeron, N. Bouchard, D. Warde-Farley, Y. Bengio, Theano: new features and speed improvements, arXiv preprint arXiv:1211.5590.

[50] S. Dieleman, J. Schlüter, C. Raffel, E. Olson, S. K. Sønderby, D. Nouri, et al., Lasagne: First release. (Aug. 2015). doi:10.5281/zenodo.27878.

URL http://dx.doi.org/10.5281/zenodo.27878

[51] D. C. Adams, E. Otárola-Castillo, geomorph: an r package for the collection and analysis of geometric morphometric shape data, Methods in Ecology and Evolution 4 (4) (2013) 393-399. doi:10.1111/2041-210X.12035 URL https://bes journals . onlinelibrary .wiley .com/doi/abs/10.1111/2041-210X.12035

[52] C. Goodall, Procrustes Methods in the Statistical Analysis of Shape, Journal of the Royal Statistical Society: Series B (Methodological) 53 (2) (1991) 285-321. doi:10.1111/j.2517-6161.1991.tb01825.x. URL https://rss.onlinelibrary.wiley.com:443/doi/abs/10.1111/j.2517-6161.1991.tb01825.x

[53] F. J. Rohlf, M. Corti, Use of two-block partial least-squares to study covariation in shape, Syst. Biol. 49 (4) (2000) 740-753.

[54] E. S. Olivas, Handbook of Research on Machine Learning Applications and Trends: Algorithms, Methods, and Techniques: Algorithms, Methods, and Techniques, IGI Global, 2009.

[55] J. Yosinski, J. Clune, Y. Bengio, H. Lipson, How transferable are features in deep neural networks?, in: Advances in neural information processing systems, 2014, pp. 3320-3328.

[56] J. Deng, W. Dong, R. Socher, L.-J. Li, K. Li, L. Fei-Fei, Imagenet: A large-scale hierarchical image database, in: 2009 IEEE conference on computer vision and pattern recognition, Ieee, 2009, pp. 248-255.

[57] K. Simonyan, A. Zisserman, Very deep convolutional networks for large-scale image recognition, arXiv preprint arXiv:1409.1556.

[58] S. Lin, Z. Zhao, F. Su, Homemade ts-net for automatic face recognition, in: Proceedings of the 2016 ACM on International Conference on Multimedia Retrieval, ACM, 2016, pp. 135-142.

[59] M. L. Collyer, D. J. Sekora, D. C. Adams, A method for analysis of phenotypic change for phenotypes described by high-dimensional data, Heredity 115 (4) (2015) 357-365. doi:10.1038/hdy.2014.75

URL http://www.nature.com/doifinder/10.1038/hdy.2014.75

[60] S. Palaniswamy, N. Thacker, C. Klingenberg, Automatic identification of landmarks in digital images, IET Computer Vision 4 (4) (2010) 247. doi:10.1049/iet-cvi.2009.0014.

URL http://digital-library.theiet.org/content/journals/10.1049/iet-cvi.2009.0014 
[61] C. Cintas, M. Quinto-Sánchez, V. Acuña, C. Paschetta, S. de Azevedo, C. Cesa r Silva de Cerqueira, V. Ramallo, C. Gallo, G. Poletti, M. C. Bortolini, S. Canizales-Qui nteros, F. Rothhammer, G. Bedoya, A. Ruiz-Linares, R. Gonzalez-José, C. o. Delrieux, Automatic ear detection and feature extraction using Geometric Morphometrics and convolutional neural networks, IET Biometrics 6 (3) (2017) 211-223. doi:10.1049/iet-bmt.2016.0002

URL http://digital-library.theiet.org/content/journals/10.1049/iet-bmt.2016.0002

[62] R. Vandaele, J. Aceto, M. Muller, F. Péronnet, V. Debat, C.-W. Wang, C.-T. Huang, S. Jodogne, P. Martinive,

P. Geurts, R. Marée, Landmark detection in 2d bioimages for geometric morphometrics: a multi-resolution tree-based approach, Scientific Reports 8 (1). doi:10.1038/s41598-017-18993-5.

URL http://www .nature.com/articles/s41598-017-18993-5

[63] X. Dai, H. Zhao, T. Liu, D. Cao, L. Xie, Locating Anatomical Landmarks on 2d Lateral Cephalograms Through Adversarial Encoder-Decoder Networ ks, IEEE Access 7 (2019) 132738-132747. doi:10.1109/ACCESS.2019. 2940623 .

[64] M. Martineau, D. Conte, R. Raveaux, I. Arnault, D. Munier, G. Venturini, A survey on image-based insect classification, Pattern Recognition 65 (2017) 273-284. doi:10.1016/j.patcog.2016.12.020. URL https://linkinghub.elsevier.com/retrieve/pii/S0031320316304411

[65] T. Elsken, J. H. Metzen, F. Hutter, Neural Architecture Search: A Survey, Journal of Machine Learning Research 20 (55) (2019) 1-21.

[66] H. Tatsuta, K. H. Takahashi, Y. Sakamaki, Geometric morphometrics in entomology: Basics and application

口 $\quad$ s: Geometric morphometrics in entomology, Entomological Science 21 (2) (2018) 164-184. doi:10.1111/ens. 12293 .

URL http://doi.wiley.com/10.1111/ens.12293

[67] R. Thevenoux, L. Folcher, M. Esquibet, D. Fouville, J. Montarry, E. Grenier, The hidden diversity of the potato cyst nematode globodera pallida in the south of peru, Evolutionary applications 13 (4) (2020) 727-737. 